OPEN ACCESS

Edited by:

Paloma Moncaleán,

Neiker Tecnalia, Spain

Reviewed by:

Yingfang Zhu,

Henan University, China Xiaoyun Li,

South China Normal University, China Akhtar Ali,

Konkuk University, Korea

*Correspondence: Victor P. Bulgakov

bulgakov@biosoil.ru

Specialty section: This article was submitted to Crop and Product Physiology, a section of the journal

Frontiers in Plant Science

Received: 04 November 2021 Accepted: 23 November 2021 Published: 03 January 2022

Citation:

Bulgakov VP and Koren OG (2022) Basic Protein Modules Combining Abscisic Acid and Light Signaling in Arabidopsis.

Front. Plant Sci. 12:808960. doi: 10.3389/fpls.2021.808960

\section{Basic Protein Modules Combining Abscisic Acid and Light Signaling in Arabidopsis}

\author{
Victor P. Bulgakov* and Olga G. Koren \\ Department of Biotechnology, Federal Scientific Center of the East Asia Terrestrial Biodiversity, Far Eastern Branch of the \\ Russian Academy of Sciences, Vladivostok, Russia
}

It is generally accepted that plants use the complex signaling system regulated by light and abscisic acid (ABA) signaling components to optimize growth and development in different situations. The role of ABA-light interactions is evident in the coupling of stress defense reactions with seed germination and root development, maintaining of stem cell identity and stem cell specification, stem elongation and leaf development, flowering and fruit formation, senescence, and shade avoidance. All these processes are regulated jointly by the ABA-light signaling system. Although a lot of work has been devoted to ABA-light signal interactions, there is still no systematic description of central signaling components and protein modules, which jointly regulate plant development. New data have emerged to promote understanding of how ABA and light signals are integrated at the molecular level, representing an extensively growing area of research. This work is intended to fill existing gaps by using literature data combined with bioinformatics analysis.

\footnotetext{
Keywords: ABA signaling, Arabidopsis, light signaling, interactome, protein modules, stress response, growth and development
}

\section{INTRODUCTION}

Several years ago, Lau and Deng (2010) summarized data on the interaction of light signaling pathways and phytohormones and noted that phytochrome-interacting factors PIF3, PIF4, PIF1/PIL5, and HY5 (long hypocotyls 5) are main light signaling components that link light signals to the signaling of phytohormones. At this time, the role of abscisic acid (ABA) signaling in light response was still poorly understood. Presently, many aspects of the interaction of light and $\mathrm{ABA}$ signaling were considered, in particular $\mathrm{ABA}$ involvement in the circadian clock (Seung et al., 2012), flowering (Park et al., 2016), guard cell sensory system (Assmann and Jegla, 2016), chlorophyll metabolism (Zhu et al., 2017), shade avoidance (Courbier and Pierik, 2019), flavonoid biosynthesis (Brunetti et al., 2019), and seedling development (Yadukrishnan and Datta, 2021). These articles mainly describe interactions of the light signaling system with ABI5 (protein abscisic acid-insensitive 5), an important signaling hub in the ABA pathway.

Although many articles consider particular aspects of cooperation between ABA and light signaling, there are no works that summarize all protein modules involved in the cooperation. For the description of the ABA-light signaling system in Arabidopsis thaliana (Arabidopsis), we used a systematic approach based on the analysis of literature data, databases, and analysis of protein interaction networks. By analyzing these data, we determined 
which Arabidopsis protein modules connect $\mathrm{ABA}$ and light signaling components. We created an integral picture of known interactions but left aside the signaling of phototropins, cryptochromes, and ultraviolet-B receptor UVR8 because their modules are still poorly resolved. Brassinosteroid (BR) and ethylene (ET) signaling were not considered in this work, although they participate in the regulation of ABAlight interplay. The reason for this is that the combined system BR-ET-ABA-light is very cumbersome to visualize and difficult to understand. Thus, this article describes only ABA-light interactions. We concentrated mainly on the balance between stress reactions and growth mediated jointly by the ABA-light signaling system. A recent review article by Yadukrishnan and Datta (2021) focused on ABA and light signaling in early seedling development. The authors noted that light causes a counteractive effect on ABA signaling, but their interaction cannot be considered as opposing each other. We are also discussing these aspects. Protein names are presented as recommended by the UniProtKB database.

\section{ABA SIGNALING}

In non-stress conditions, $\mathrm{ABA}$ plays a dual role, acting as an inhibitor or activator of plant growth and development. ABA promotes hypocotyl elongation in the dark, promotes leaf and root growth as well as inhibiting stomatal transpiration and leaf initiation (Yoshida et al., 2019). ABA under non-stress conditions is important for correct stomatal development and floral transition (Yoshida et al., 2019). Under stress conditions, $\mathrm{ABA}$ controls stomatal aperture, thereby reducing $\mathrm{CO}_{2}$ entry into leaves and limiting photosynthesis and increasing tolerance to stress conditions (Dong et al., 2015; Kuromori et al., 2018). Endogenous basal ABA does not directly suppress photosynthesis and inhibits a stress-escape response (avoidance of stress through reproduction, a response that is unfavorable for an individual plant, but necessary to preserve the species) under normal conditions, allowing plants to accumulate biomass and increase yield (Negin et al., 2019). However, long-term $\mathrm{ABA}$ treatment causes COP1 activation in a light intensity-dependent manner to suppress chloroplast development and balance growth and stress responses (Lee et al., 2021). PhyB stimulates the ABA biosynthesis in the shoots, and mobile shoot-to-root ABA signaling links phyBmediated light perception with root reactive oxygen species (ROS) homeostasis for optimal plant propagation (Ha et al., 2018).

Stress induces $A B A$ accumulation and binding to $A B A$ receptors of the PYR/PYL/RCAR family to inhibit A-type protein phosphatases 2C (PP2Cs). PP2C inactivation activates class 3 sucrose nonfermenting-1-related protein kinases (SnRK2s) that phosphorylate ABA-responsive element binding factors ( $\mathrm{ABFs}$ ). Activated $\mathrm{ABFs}$ initiate the expression of responsive genes by binding to the cis-acting ABA response element (ABRE, Fujii et al., 2009; Lumba et al., 2014). The basic scheme of ABA signaling is shown in Figure 1.

\section{BASIC PHOTOMORPHOGENETIC MECHANISMS}

The A. thaliana genome encodes five phytochrome proteins: phyA mediates responses to far-red light (FR), and phyB-phyE mediates shade avoidance and classical red light (R)/FR-reversible responses (Clack et al., 2009). In the dark, plants repress photomorphogenesis by the complexes consisting of E3 ubiquitin-protein ligase COP1 (constitutive morphogenic1) and SPA (suppressor of phytochrome A) proteins (Xu et al., 2014). An important role in the degradation of positively acting transcription factors is also played by basic helix-loop-helix (bHLH) transcription factors PIFs. In response to light, phytochromes inactivate COP1-SPA complexes and induce degradation of negative regulators of photomorphogenesis, PIFs (Figure 1). PhyB inhibits the regulatory activity of PIF1 (synonym: PIL5) and PIF3 by releasing them from DNA targets. These events promote photomorphogenesis by establishing the dynamic protein module PIF1-COP1/SPA1 and triggering HY5-mediated light signaling (Xu et al., 2014; Pham et al., 2018a; Paik et al., 2019; Yang and Liu, 2020). The COP1-SPA complexes are also important for sensing vegetative shade, which is determined as a reduction in the R:FR ratio and is essential for hypocotyl growth and leaf petiole elongation.

PIF1, PIF3, PIF4, PIF5, and PIF7 contain an active phyBbinding (APB) domain, which is necessary for interaction with light-activated phyB. PIF1 and PIF3 also bind to phyA through an additional phyA-binding (APA) domain (Legris et al., 2017). According to $\mathrm{Xu}$ et al. (2014), the bHLH domain of PIF1 interacts with the bZIP domain of HY5, and the N-terminal domain of PIF1 interacts with the WD40 repeat domain of COP1. The resulting complex promotes degradation of HY5 via the $26 \mathrm{~S}$ proteasome pathway. PIF4 is an integrator of numerous signaling pathways and optimizes growth in environmental conditions (Choi and Oh, 2016; Legris et al., 2017). PIF4 is better known as a regulator of thermosensitive growth, although its role in cold resistance is also known (Choi and Oh, 2016). The role of PIFs as critical signal integrators is discussed in recent review articles (Legris et al., 2017; Paik et al., 2017; Pham et al., 2018a,b; Favero, 2020; Jing and Lin, 2020).

\section{ABA-LIGHT INTEGRATIVE MODULES}

Figure 2 represents the joint ABA-light signaling network generated on the base of the Arabidopsis PAIR database (Lin et al., 2011).1,2,3,4,5 The ABA receptors PYR/PYL/RCAR and phyA/B were chosen as input proteins for the network. Yellow circles represent proteins of the light signaling system, which has a function in the Gene Ontology (GO) Process "Response to Abscisic Acid." Each of these proteins forms its own signaling module, described in separate subsections of the article. The most known and probably

\footnotetext{
${ }^{1}$ http://www.cls.zju.edu.cn/pair/

${ }^{2}$ http://thebiogrid.org/

${ }^{3}$ https://www.uniprot.org/

${ }^{4}$ https://www.arabidopsis.org/

${ }^{5}$ https://www.ebi.ac.uk/intact/interactors/
} 


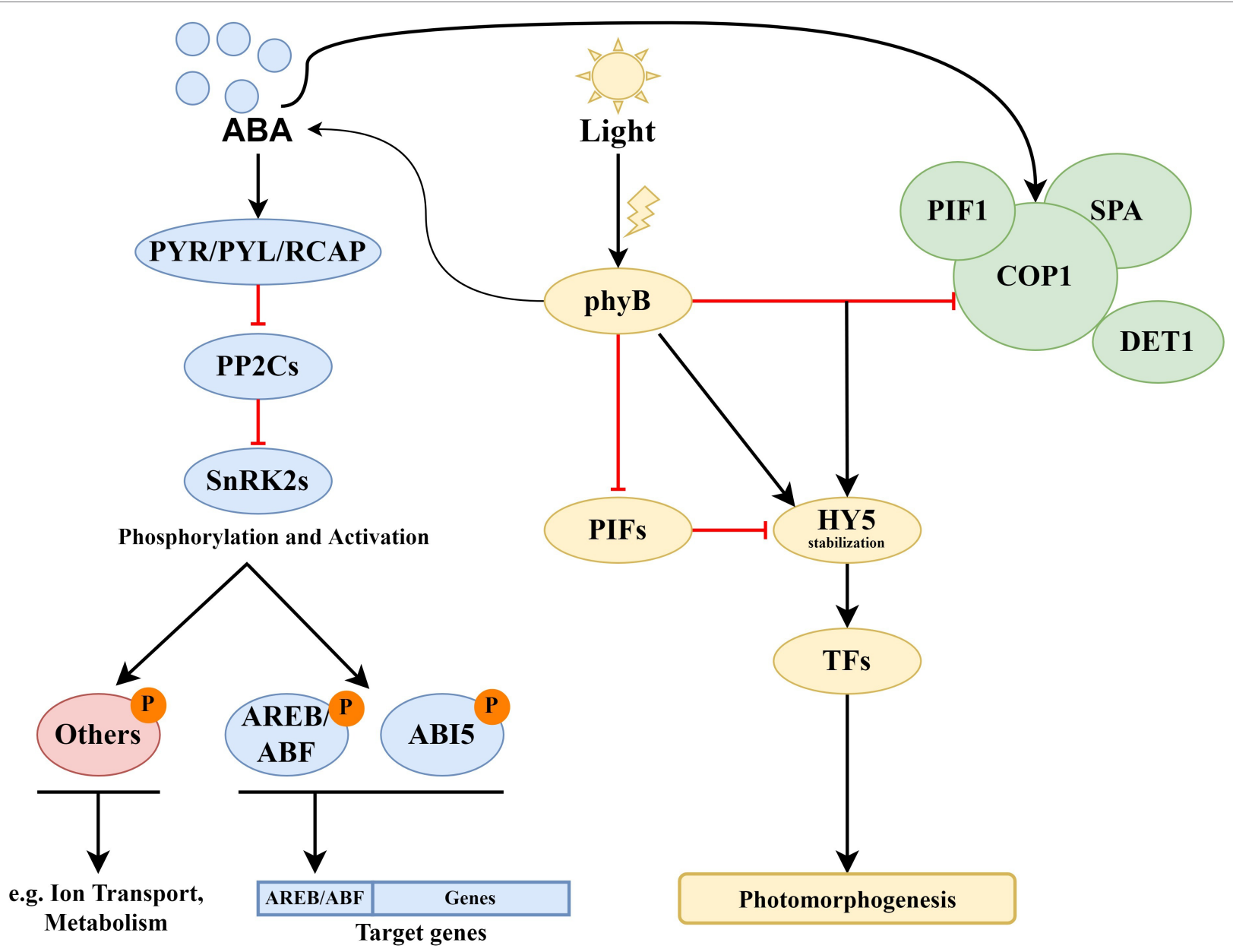

FIGURE 1 | Core components of the ABA and light signaling systems. Long-term ABA treatment causes COP1 activation and phyB stimulates the ABA biosynthesis. ABA activates ABA receptors of the PYR/PYL/RCAR family that inhibits A-type protein phosphatases 2C (PP2Cs). PP2C inactivation activates class 3 sucrose nonfermenting-1related protein kinases (SnRK2s) that phosphorylate ABA-responsive element binding factors AREB/ABF. Activated ABFs initiate the expression of responsive genes by binding to the cis-acting ABA response element (ABRE). In response to light, phytochromes inactivate COP1-SPA complexes and induce degradation of negative regulators of photomorphogenesis, PIFs. PhyB inhibits the regulatory activity of PIF1 and PIF3. These events promote photomorphogenesis by establishing the dynamic protein module PIF1-COP1/SPA1 and triggering HY5-mediated light signaling. Degradation of PIFs and stabilization of HY5 result in promotion of photomorphogenesis. HY5 physically interacts with transcription factors (TFs) and other proteins, and directly affects the expression of numerous genes. By promoting COP1 protein destabilization and turnover, DET1 positively regulates COP1 activity toward the degradation of HY5. Keeping HY5 levels tightly regulated is essential for its functioning during dark-to-light transition.

most important module is based on HY5. Far-red elongated hypocotyls 3 (FHY3) and far-red impaired response 1 (FAR1) are positive regulators of $\mathrm{ABA}$ signaling that participate in plant development, The MYB-related transcription factors LHY/CCA1 serve as a link between the circadian clock and cold response. The main role of early flowering 3 (ELF3), as a component of the Evening Complex, is to connect light signaling with thermotolerance. Flowering time control protein FCA (FCA) is involved in the ABA pathway and ROS modulation (Lee et al., 2015), and transcription factor HFR1 is involved in the response to light intensity, shade avoidance, and dark-induced senescence. The ABI3-CONSTANS (CO) interaction has a role in the maintenance of embryo dormancy (Kurup et al., 2000). These proteins and interactions between them are critical for interaction with the $\mathrm{ABA}$ signaling system. In addition, the data indicate several interactions between $\mathrm{ABA}$ and light-signaling proteins with as yet unknown physiological roles. Among them are the flowering locus T (FT)-ABF4 interaction (Braun et al., 2011), ABI3-TOC1 (Kurup et al., 2000), and interactions of ABA receptors with PIFs (Qi et al., 2020). ABA-light integrative modules and descriptions of their functional significance are presented in Table $\mathbf{1}$.

Our analysis showed that most of the described interactions between the light signaling system and ABA are aimed at regulating $\mathrm{ABA}$ signaling from the side of light signals. First of all, this is mainly the regulation of the expression of $A B I 5$, as well as $A B I 1, A B I 3$ and $A B I 4$ genes, which is carried out using the following modules: HY5 $\rightarrow A B I 5, \mathrm{BBX} 21-\| A B I 5$, phyB -\| PIF1 $\rightarrow A B I 3$ and $A B I 5$, ELF3 $-\| P I F 4 \rightarrow A B I 5$, DET1 - $\|$ FHY $\rightarrow A B I 1, A B I 3, A B I 4$ and $A B I 5$, DET1 - $\|$ $A B I 5$, and LHY $\rightarrow A B I 5$. LHY suppresses the expression of $A B I 1 / A B I 2, S n R K 2.2$, and $A B F 1$. Protein-protein interactions are realized through $\mathrm{CO}-\| \mathrm{ABI} 3$ and FCA $\rightarrow$ ABI5. 


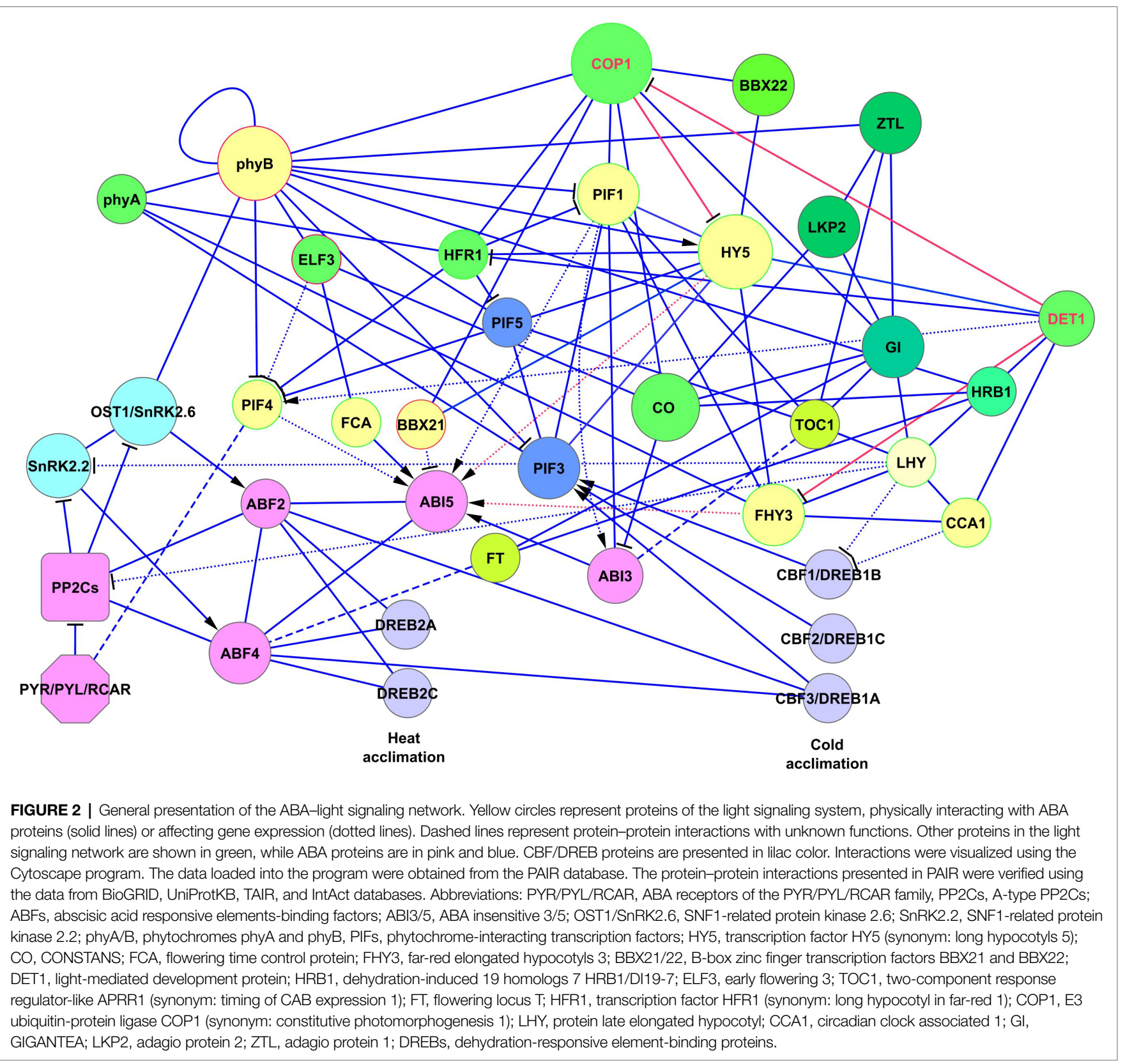

From the ABA side, the light signaling system is regulated by the ABA receptors PYL8/PYL9, which promote PIFs protein accumulation in the dark. SnRK2.6 interacts with phyB, having a negative role in red light-induced stomatal opening. ABI5 and ABI4 enhance the expression of the FLC gene.

\section{EFFECT OF LIGHT ON THE EXPRESSION OF GENES ENCODING ABA SIGNALING COMPONENTS}

A global analysis of gene expression during intense light treatment showed that ABA levels were gradually increased from 0.5 to $72 \mathrm{~h}$ due to enhanced expression of $A B A$ biosynthetic genes (Huang et al., 2019). The authors suggested the important role of ABA in middle- and long-term high light stress response, although the data on the gene expression of the ABA signaling network were somewhat surprising. Among the down-regulated genes were genes encoding $\mathrm{ABA}$ receptors: PYR1, PYL3, PYL4, PYL5, PYL7, and PYL9. PP2Cs genes were upregulated, such as $A B I 1, A B I 2, H A B 1, A H G 1$, PP2CA, and especially HAI1, HAI2, and HAI3 (Huang et al., 2019). SnRK2.9, $A B F 2$, and $A B F 3$ were slightly downregulated with different dynamics. Altogether, these data indicate silencing of ABA signal transmission. It is not yet known which mechanisms regulate the expression of the listed genes. 
TABLE 1 | ABA-light integrative modules and their functional significance.

\begin{tabular}{|c|c|c|c|c|}
\hline $\begin{array}{l}\text { Light signaling } \\
\text { protein }\end{array}$ & $\begin{array}{l}\text { ABA-responsive gene/ } \\
\text { protein }\end{array}$ & Signaling module & Function & References \\
\hline HY5 & $A B / 5$ & $\mathrm{HY} 5 \rightarrow \mathrm{AB} / 5$ & $\begin{array}{l}\text { ABA responses in seed germination, } \\
\text { seedling growth, and root development } \\
\mathrm{H}_{2} \mathrm{O}_{2} \text { production via the activation of } \\
\mathrm{NADPH} \text { oxidase. Tolerance to excess light } \\
\text { and cold }\end{array}$ & $\begin{array}{l}\text { Chen et al., } 2008 \\
\text { Zhou et al., 2014; Wang } \\
\text { et al., } 2018\end{array}$ \\
\hline $\mathrm{BB} \times 21$ & $A B / 5$ & $\mathrm{BBX} 21-\| A B / 5$ & Gas exchange in drought conditions & $\begin{array}{l}\text { Xu et al., 2014; Kang et al., } \\
2018\end{array}$ \\
\hline PIF1 & $A B / 3$ and $A B / 5$ & phyB $-\| \mathrm{PIF} 1 \rightarrow A B / 3$ and $A B / 5$ & $\begin{array}{l}\text { Increased ABA responsiveness, inhibition } \\
\text { of seed germination }\end{array}$ & Oh et al., 2009 \\
\hline PIF1 PIF4 & $A B / 5$ & ELF3 $-\|$ PIF4 $\rightarrow$ ABI5 & $\begin{array}{l}\text { Leaf senescence at high ambient } \\
\text { temperature }\end{array}$ & $\begin{array}{l}\text { Sakuraba et al., 2014; Kim } \\
\text { et al., } 2020\end{array}$ \\
\hline PIF1 PIF3 PIF4 PIF5 & PYL8/PYL9 & $\begin{array}{l}\text { PYL8/PYL9 } \rightarrow \text { PIF4 } \\
\text { PYL8/PYL9 }-\| \text { PIF4 } \rightarrow \text { ABI5 }\end{array}$ & $\begin{array}{l}\text { PYL8 and PYL9 promote PIF4 protein } \\
\text { accumulation in the dark and enhance } \\
\text { PIF4 binding to the } A B I 5 \text { promoter, but } \\
\text { negatively regulate PIF4-mediated } A B / 5 \\
\text { activation }\end{array}$ & Qi et al., 2020 \\
\hline PIF3 PIF5 & $\begin{array}{l}\text { PYL3, PYL6, PYL12, } \\
\text { SnRK2.2, ABI5 and ABF3 }\end{array}$ & $\begin{array}{l}\text { Potential targets indicated (further } \\
\text { study of gene expression required) }\end{array}$ & Unknown & Liang et al., 2020 \\
\hline FHY3 & $A B / 1, A B / 3, A B / 4$ and $A B / 5$ & $\begin{array}{l}\mathrm{DET} 1-\| \mathrm{FHY} 3 \rightarrow A B / 1, A B / 3 \\
A B / 4 \text { and } A B / 5\end{array}$ & $\begin{array}{l}\text { Activation of the ABA pathway after the } \\
\text { dark-to-light transition. Pathway } \\
\text { repressor: DET1 }\end{array}$ & Xu et al., 2020 \\
\hline DET1 & $A B / 5$ & $\begin{array}{l}\text { DET1 - } \| A B / 5 \text { (via recruiting } \\
\text { HDA6 to } A B / 5 \text { promoter) }\end{array}$ & $\begin{array}{l}\text { Positive regulation of light-induced } \\
\text { greening; epigenetic control of } A B / 5 \\
\text { expression }\end{array}$ & Xu et al., 2020 \\
\hline $\mathrm{CO}$ & $A B / 3$ & $\mathrm{CO}-\| \mathrm{ABI} 3$ & $\begin{array}{l}\text { Quiescence in developing seedlings, rapid } \\
\text { induction of flowering }\end{array}$ & Kurup et al., 2000 \\
\hline phyB & SnRK2.6 & SnRK2.6-phyB & $\begin{array}{l}\text { Negative role of SnRK2.6 in red light- } \\
\text { induced stomatal opening }\end{array}$ & Li et al., 2021 \\
\hline FCA & $\mathrm{ABI} 5$ & $\mathrm{FCA} \rightarrow \mathrm{ABI} 5$ & $\begin{array}{l}\text { Adaptation of embryo and flower } \\
\text { development to stress-induced ROS, } \\
\text { heat, cold, and drought conditions }\end{array}$ & Lee et al., 2015 \\
\hline \multirow[t]{4}{*}{ LHY } & $A B / 1, A B / 2$ & $\mathrm{LHY}-\| A B / 1 / 2$ & $\begin{array}{l}\text { Coupling between the circadian clock and } \\
\text { ABA pathways }\end{array}$ & \multirow[t]{4}{*}{ Adams et al., 2018} \\
\hline & $\operatorname{SnRK2.2}$ & LHY - \| SnRK2.2 & Activation of drought-tolerance processes & \\
\hline & $A B F 1$ & LHY $-\| A B F 1$ & $\begin{array}{l}\text { Potentiation of } A B A \text { responses in the } \\
\text { morning }\end{array}$ & \\
\hline & $A B / 5$ & $\mathrm{LHY} \rightarrow A B / 5$ & $\begin{array}{l}\text { Responses to unexpectedly hot or dry } \\
\text { conditions in the daytime }\end{array}$ & \\
\hline \multirow[t]{2}{*}{ FLC } & $\mathrm{ABI} 5$ & $\begin{array}{l}\text { SnRK2s } \rightarrow \text { ABI5 (and/or other } \\
\text { ABFs) } \rightarrow \text { FLC }\end{array}$ & Inhibitory effect on floral transition & Wang et al., 2013 \\
\hline & $\mathrm{AB} \mid 4$ & $\mathrm{AB} \mid 4 \rightarrow F L C$ & & Shu et al., 2016 \\
\hline
\end{tabular}

A recent work by Qi et al. (2020) contributed to this problem, as they showed that PIFs physically interact with the ABA receptors PYL8 and PYL9. These authors showed that PYL8 and PYL9 negatively regulate PIF4-mediated trans-activation of ABI5. Liang et al. (2020) analyzed the DNA-binding sites for PIF3 and showed that several ABA signaling genes were found to be potential targets, such as PYL3, PYL6, PYL12, SnRK2.2, $A B I 5$, and $A B F 3$ (Table 1).

The complexity of the ABA-light interaction was noticed by $\mathrm{Oh}$ et al. (2009) who found that responsiveness to ABA is due to the summed activity of both positively and negatively regulated genes of the ABA signaling pathway. The view that the ABA-light collaboration is a complex and finely tuned process was also supported by Qi et al. (2020).

\section{HY5-BASED MODULES}

HY5 is a transcription factor that promotes photomorphogenesis in light, acting downstream of the light receptor network and directly activating the transcription of light-induced genes. Among these are genes encoding transcription factors, signaling proteins, and flavonoid biosynthetic enzymes (Gangappa and Botto, 2016; Cañibano et al., 2021). PhyB-dependent induction of HY5 is well known (Guo et al., 2021). The activation of HY5 by phyB may take place directly by physical interaction (Chai et al., 2015) or through intermediaries (Ortigosa et al., 2020). HY5 forms a complex network with proteins of the light signaling system, such as COP1, PIF3, FHY3, FAR1, HFR1, light-mediated development protein DET1, and B-box binding (BBX) proteins (BioGrid annotation). 
The important effect of cooperation between ABA and light is the decision to transition from a dormant seed to photoautotrophic growth. It is well documented that the interaction of light signaling components with ABA signaling is necessary to fine-tune seed germination and seedling development, and this interaction occurs via signaling on the $A B I 5$ promoter (Chen et al., 2008). HY5 binds to the $A B I 5$ promoter and activates its expression, thus mediating ABA responses in seed germination, seedling growth, and root development (Chen et al., 2008). HY5 is an important hub in the crosstalk between light and cold response pathways, and an integrator of ABA and ROS signaling (Wang et al., 2018). HY5 is a positive regulator of cold acclimation; in particular, increased expression of HY5 induces increased expression of cold-responsive (COR) genes (Catalá et al., 2011). The authors proposed a model in which HY5 would promote the full development of cold acclimation, integrating low temperature and light signaling. According to this model, HY5 is stabilized by cold by protein stabilization through the nuclear depletion of COP1 (Catalá et al., 2011). HY5 also induces several genes involved in anthocyanin biosynthesis (Gangappa and Botto, 2016).

The current model suggests that ROS production and scavenging pathways are integrated into a signal transduction network, which is necessary for stress acclimation (Mittler et al., 2011; Devireddy et al., 2020). In tomato, ABA signaling realized through $A B I 5$ induces $\mathrm{H}_{2} \mathrm{O}_{2}$ production via the activation of respiratory burst oxidase homolog 1 (SlRboh1; Zhou et al., 2014; Wang et al., 2018). This mechanism includes binding HY5 to the $A B I 5$ promoter and ABI5-inducing $R B O H 1$ expression (Wang et al., 2018), establishing the signaling sequence HY $5 \rightarrow A B I 5 \rightarrow R b o h 1$. A similar mechanism is likely to function in Arabidopsis. Therefore, through binding to the promoter of $A B I 5$, HY5 triggers enhanced photoprotection through the induction of an apoplastic $\mathrm{H}_{2} \mathrm{O}_{2}$ burst that influences antioxidant status, cyclic electron flux, and nonphotochemical quenching. Improved photoprotection allows shaded leaves to better tolerate photoinhibition. The HY5-ABI5 pathway is necessary to ensure tolerance to excess light and cold (Gangappa and Botto, 2016).

HY5 and its homolog HYH, collectively with PIF1 and PIF3 form a dynamic activation-suppression transcriptional module HY5/HYH-PIF1/PIF3 to establish an appropriate cellular redox status in the response to light (Chen et al., 2013; ToledoOrtiz et al., 2014). PIF1/PIF3 and HY5/HYH function antagonistically; they physically interact and jointly regulate the expression of ROS-responsive genes (Chen et al., 2013). The author's model shows that in the dark, PIF1 and PIF3 accumulate while HY5 and HYH are degraded. In high light conditions, photosensitized Protochlorophyllide (Pchlide) generates singlet oxygen, which causes photooxidative cell death. However, light promotes the stabilization of $\mathrm{HY} 5 / \mathrm{HYH}$ and PIF1/PIF3 degradation, causing activation the ROS signaling and the expression of ROS responsive genes, thus adjusting ROS level and allowing plants to survive under light stress conditions (Chen et al., 2013). Besides HY5/HYH-PIF1/PIF3 module-mediated control of ROS-responsive genes, these genes can be controlled by HY5 and other PIFs (PIF4 and PIF5) in association with circadian clock components (Toledo-Ortiz et al., 2014). HY5, PIF4, and PIF5 proteins are under strong diurnal regulation, while PIF1 and PIF3 are not rhythmic (Toledo-Ortiz et al., 2014). The link between HY5/PIF modules and the ABA signaling system is realized through regulation of $A B I 3$ and $A B I 5$ expression: $\mathrm{HY} 5 \rightarrow A B I 5, \mathrm{PIF} 1 \rightarrow A B I 3 / A B I 5$, and PIF $4 \rightarrow A B I 5$ (Table 1).

\section{SIGNALING ON THE ABI5 PROMOTER}

According to TAIR annotation, ABI5 participates in the ABA-activated signaling pathway, negative regulation of seed germination, positive regulation of transcription, responses to chitin, gibberellin, salt stress, water deprivation, seed development, seed germination, and the sugar-mediated signaling pathway. $A B I 5$ encodes the basic leucine zipper transcription factor involved in ABA signaling. The Arabidopsis ABA-insensitive abi5 mutants have pleiotropic defects in ABA response, including altered expression of ABA-regulated genes and decreased sensitivity to ABA inhibition of germination. ABI5 regulates a subset of late-embryogenesis-abundant genes during seed development and vegetative growth.

G-box cis-elements of the $A B I 5$ promoter are binding sites for HY5, BBX21, and PIF1, whereas FHY3 and FAR1 bind FBS cis-elements (CACGCGC) on ABI5 (Xu et al., 2014; Ma and $\mathrm{Li}, 2018$ ). BBX21, also known as $\mathrm{STH} 2$, is a BBX protein, which binds to the $A B I 5$ promoter and negatively regulates ABI5 expression (Xu et al., 2014; Kang et al., 2018). In the BBX protein subfamily, BBX21/STH2 and BBX22/STH3 (also known as LZF1) function as positive regulators of photomorphogenesis, whereas BBX18/DBB1a, BBX19/DBB1b, $\mathrm{BBX} 24 / \mathrm{STO}$, and $\mathrm{BBX} 25 / \mathrm{STH} 1$ are negative regulators (Xu et al., 2014). Two light signaling proteins, HRB2 (CHD3-type chromatin-remodeling factor PICKLE) and BBX21 repress ABA signaling by $A B I 5$ suppression to sustain gas exchange in drought conditions (Kang et al., 2018). Mutations in HRB2 or $B B X 21$ cause ABA hypersensitivity and reduced water loss (Kang et al., 2018). Accordingly, plants overexpressing BBX21 showed reduced sensitivity to ABA and improved photosynthetic capacity under drought conditions (Gómez-Ocampo et al., 2021). Interactions of ABI5 with the ABA-light signaling network are shown in Figure 3. The interaction of HY5, DET1, and FHY3 signaling with ABI5 is described in more detail in the next section.

\section{FHY3/FAR1 AND DET1 IN THE PROCESS OF INTERACTION WITH ABA SIGNALING}

Far-red elongated hypocotyl (FHY3) and far-red impaired response 1 (FAR1) are positive regulators of ABA signaling because they bind to the $A B I 5$ promoter and activate its transcription, thus mediating abiotic stress responses and $\mathrm{ABA}$ signal transduction (Tang et al., 2013; Ma and Li, 2018). Disruption of FHY3 and/or FAR1 decreases the sensitivity to 


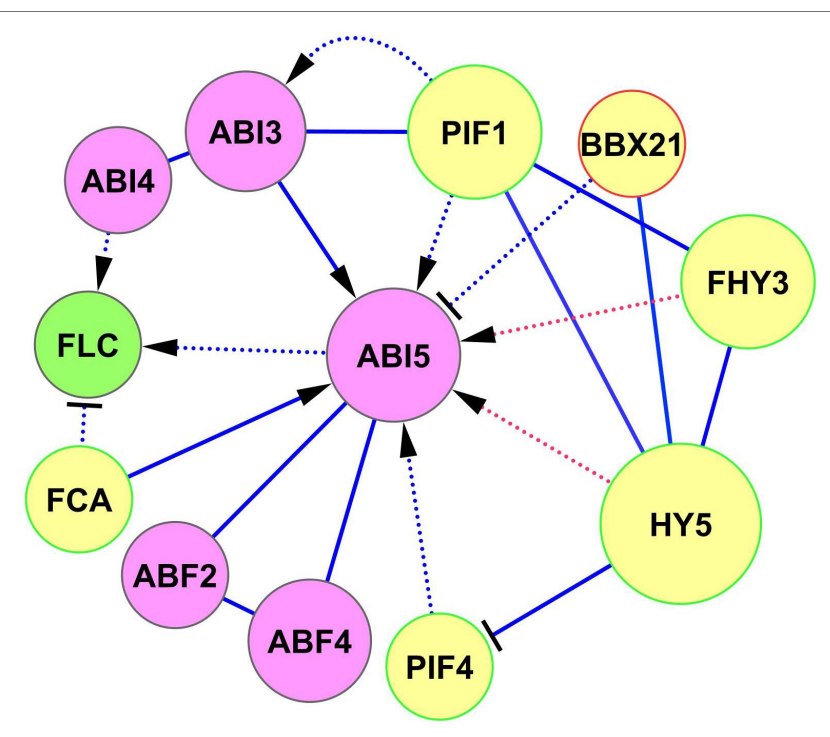

FIGURE 3 | Crosslinking of ABA and light signaling on ABI5. Solid lines represent protein-protein interactions presented in PAIR, BioGRID, and IntAct, and dotted lines represent transcriptional regulation. ABA-responsive element binding factors of the ABA signaling network, such as ABF2, ABF4, and $\mathrm{ABI} 3$ (involved in abiotic stress defense and stress memory) cooperate with $\mathrm{ABI} 5$ to jointly regulate its function. Light signaling proteins PIF1, PIF4, FHY3/FAR1, and HY5 activate the expression of $A B / 5$, whereas BBX21 inhibits its expression. FCA activates $A B I 5$ by protein-protein interaction and jointly with $\mathrm{ABI} 5$ regulates expression of FLC. FLC and FCA are proteins that substantially reduce plant water use and are important for heat and cold adaptation. Red activator lines from FHY3/FAR1 and HY5 indicate cooperative effects HY5, DET1, and FHY3 signaling on ABI5 function (see also details in Figure 4). Abbreviations: $A B I 3,4,5$, ABA insensitive 3,4,5; ABFs, abscisic acid responsive elements-binding factors; PIFs,

phytochrome-interacting transcription factors; HY5, transcription factor HY5 (protein long hypocotyls 5); BBX21, B-box zinc finger transcription factor BBX21; FHY3/FAR1, far-red elongated hypocotyls 3/protein far-red impaired response 1; FCA, flowering time control protein; FLC, MADS-box protein flowering locus $\mathrm{C}$.

ABA-mediated inhibition of seed germination, seedling development, and primary root growth (Tang et al., 2013). The fhy 3 and far 1 mutants have wider stomata, lose water faster, and are more sensitive to drought than the wild type. FAR1 negatively regulates light-induced ROS accumulation and oxidative stress-induced cell death by activating the transcription of myo-inositol-1-phosphate synthase 1 (MIPS1; Ma and Li, 2018).

In addition to its well-known function in plant growth and development during the vegetative stage, FHY3 plays an essential role in floral meristem determinacy and shoot apical meristem maintenance (Li et al., 2016). FHY3 directly represses CLAVATA 3 in shoot apical meristem, and consequently regulates WUSCHEL to maintain the stem cell pool. FHY3 mainly acts as a transcriptional repressor in flower development, in contrast to its activator role in seedlings, i.e., its function varies in different organs (Li et al., 2016). Why FHY3 acts as either activator or repressor in different organs or developmental stages remains to be studied ( $\mathrm{Ma}$ and $\mathrm{Li}, 2018$ ).

The role of FHY3 in the light-ABA signaling is complex and depends on the activity of the light-mediated development protein DET1 (DET1), which is an important modulator of the light signaling system (Xu et al., 2020). During seedling development, DET1 physically interacts with FHY3 and represses FHY3-mediated activation of ABA-responsive genes. Among these genes are $A B I 1, A B I 3, A B I 4$, and $A B I 5$ (Xu et al., 2020). The authors tested these genes as markers of the ABA-activated pathway. Interestingly, while ABI3, ABI4, and ABI5 are transcription factors, $\mathrm{ABI} 1$ is a protein phosphatase. ABI5 acts as a central hub for these interactions (Yadukrishnan and Datta, 2021). The DET1 - $\|$ FHY3 $\rightarrow$ ABI5 pathway acts only in light conditions. The role of this pathway is that after the dark-to-light transition, ABA induces high expression of $A B I 5$. This response is balanced and adjusted by DET1 through FHY3 repression that causes the greening of etiolated seedlings and increases their adaptation to light. COP1 and PIFs were proposed could act collectively with DET1 and FHY3 to prevent the activity of the signaling module DET1 - $\|$ FHY3 $\rightarrow$ ABI5 in darkness (Xu et al., 2020). The crossing of HY5, DET1, and FHY3 signaling with $A B I 5$ is presented in Figure 4.

The important role of DET1 is its involvement in epigenetic control, suggesting a role in the generation of stress memory. DET1 physically interacts with histone deacetylase 6 (HDA6), recruiting HDA6 to the $A B I 5$ promoter (Xu et al., 2020). This inhibits the activation of $A B I 5$ by regulating the enrichment of $\mathrm{H} 3 \mathrm{~K} 27 \mathrm{ac}$ and $\mathrm{H} 3 \mathrm{~K} 4 \mathrm{me} 3$ modifications. This mechanism can be independent of FHY3 and contributes to the stress memory.

DET1 is also involved in ABA responses by interacting with ABI3 (Kurup et al., 2000). The consequences and outputs of this interaction are yet unknown. Because DET1 and COP1 are repressors of photomorphogenesis and repressors of multiple light responses, destruction of any of these proteins results in the induction of de-etiolation in darkness and hyper-photomorphogenic phenotype in the light. DET1 is associated with COP1 in vivo (Cañibano et al., 2021), and DET1/COP1 and HY5 are now considered as two main distinct signaling components (albeit with some overlap of function) in the regulation of thermosensory elongation (Xu et al., 2020). The DET1/COP1 association promotes PIF4 expression and stabilizes PIF4 protein (Gangappa and Kumar, 2017, 2018). The author's model suggests that the DET1/COP1 $\rightarrow$ PIF4 signaling sequence acts as an important module for the control of growth and plant stress defense in response to seasonal signals. They showed that DET1/COP1-activated PIF4 binds to the promoters of growth genes and activates their expression, whereas HY5 negatively regulates thermosensory growth by competing with PIF4. Elevated temperatures decrease HY5 activity and activate PIF4 target genes (Gangappa and Kumar, 2017).

DET1, via interaction with COP1, decreases the abundance of HY5, thus avoiding hyper-photomorphogenic responses (Cañibano et al., 2021). These authors proposed an interesting mechanism according to which these proteins control HY5 abundance. They postulated that DET1-mediated COP1 degradation is necessary to maintain COP1 turnover and activity. Indeed, COP1 is a short-lived protein with a high turnover rate. By down-regulating HY5 levels, DET1 prevents HY5 


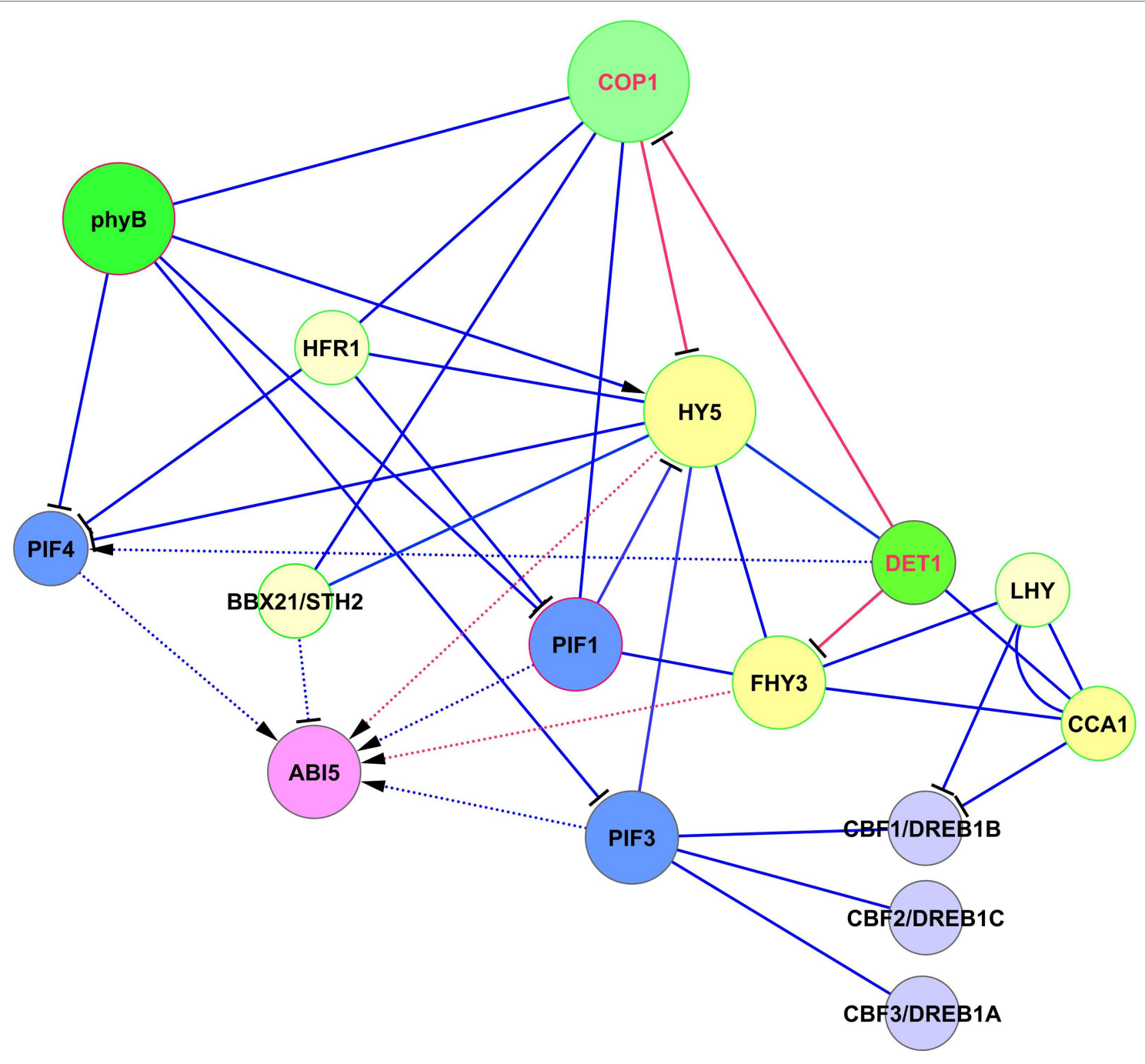

FIGURE 4 | Crossing of HY5, DET1, and FHY3 signaling with ABI5 function. ABI5 expression is regulated in two ways. The first one represents activation of $A B / 5$ expression by HY5 by the DET1 $-\|\mathrm{COP} 1-\| \mathrm{HY} 5 \rightarrow A B / 5$ module. The second level of regulation is realized by parallel pathway via DET1 $-\| \mathrm{FHY} 3 \rightarrow A B I 5$ and $\mathrm{DET} 1 / \mathrm{COP} 1 \rightarrow \mathrm{PIF} 4 \rightarrow A B / 5$ modules, where DET1/COP1 association promotes PIF4 expression and stabilizes PIF4 protein. DET1 also can form a complex with the CCA1/LHY module. Abbreviations: ABI5, ABA insensitive 5; phyA/B, phytochromes phyA and phyB; PIFs, phytochrome-interacting transcription factors; HY5, transcription factor HY5; FHY3, far-red elongated hypocotyls 3; BBX21, B-box zinc finger transcription factor BBX21; DET1, light-mediated development protein; CCA1, CCA1, circadian clock associated 1; LHY, late elongated hypocotyl; HFR1, transcription factor HFR1; COP1, E3 ubiquitin-protein ligase COP1; CBFs/ DREBs, dehydration-responsive element-binding proteins.

binding to primary targets. Since HY5 behaves in light conditions mainly as a transcriptional activator, DET1's function is necessary to prevent HY5 over-accumulation that causes misregulation of numerous downstream genes. HY5 transcriptional activity over a limited gene number can be regulated by titration of its availability (Cañibano et al., 2021), resembling the process known from animal studies, where signaling molecules form spatiotemporal concentration gradients that respond to a wide range of signal intensities, the so-called digital process (Tay et al., 2010). Thus, DET1 - $\|$ FHY3 $\rightarrow$ ABI5 and DET1/ $\mathrm{COP} 1 \rightarrow \mathrm{PIF} 4 \rightarrow A B I 5$ modules emerge as important players in the regulation of development and stress reactions, in addition to HY5-based modules.

\section{LHY/CCA1 AS A LINK BETWEEN THE CIRCADIAN CLOCK AND STRESS RESPONSE}

LHY and CCA1 bind to the same region of gene promoters, but they are only partially redundant (UniProtKB annotation). The CCA1 protein responds to environmental light and temperature signals and, therefore, may maintain circadian rhythms as well as adapting plants to the environment (Zhang et al., 2021).

Adams et al. (2018) suggested a mechanism for the circadian control of ABA accumulation in plants. ABA accumulates 
during water deficit, reaching its peak in the evening. In the morning, LHY inhibits the expression of ABA biosynthetic genes encoding 9-cis-epoxycarotenoid dioxygenase enzymes that decreases ABA level and ensures the rhythmic accumulation of ABA. LHY also controls ABA signaling pathway by inhibiting the expression of the $A B I 1, A B I 2, S n R K 2.2$, and $A B F 1$ but activating the expression of $A B I 5$ (Adams et al., 2018). The resulting effect of LHY is that under physiologically relevant conditions, LHY acts to potentiate ABA-dependent stress responses by promoting the expression of ABA-responsive genes involved in establishing increased tolerance to drought and osmotic stress.

Although LHY and CCA1 are almost identical within their DNA-binding domains, they have functional differences. The most represented motif for LHY and CCA1 is the EE motif (AAATATCT or AGATATTT). However, the ABRE motif (ABA-specific motif) was over-represented only in LHY-specific target promoters, but not in CCA1-specific target promoters (Adams et al., 2018). Commenting on this article, Belbin and Dodd (2018) noted that there is some overlap in the roles of LHY and CCA1 in regulating ABA signaling. It is interesting that DET1 forms a complex with LHY/CCA1 to repress two-component response regulator-like APRR1/TOC1 expression in the morning (Paik et al., 2017), which adds an extra level of regulation to the above described DET1 $-\|$ FHY3 $\rightarrow$ ABI5 and DET1/COP $1 \rightarrow \mathrm{PIF} 4 \rightarrow A B I 5$ modules.

Thus, both LHY and CCA1 transcription factors, acting in conjunction with the ABA signaling system, regulate the adaptation of plants to cold, high temperature, lack of water, and osmotic stress, thereby optimizing defense responses under circadian rhythms.

\section{HFR1'S ROLE IN SHADE AVOIDANCE AND DARK-INDUCED SENESCENCE}

An important player in shade avoidance and dark-induced senescence is transcription factor HFR1 (long hypocotyl in FR light1), which is the non-DNA binding atypical HLH factor (Rolauffs et al., 2012; Pham et al., 2018b; Ueda et al., 2020). Like FHY3, HFR1 is involved in FR light signal transduction and physically interacts with phyA (IntAct and STRING databases). According to GO annotation, HFR1 is involved in the ABA-activated signaling pathway. However, HFR1 does not interact with any component of the ABA signaling pathway but interacts with PIF1, PIF3, PIF4, and PIF5 (BioGRID). Probably, the involvement in the ABA signaling pathway occurs via PIFs. PIF4 and PIF5 are required for shade avoidance and dark-induced senescence acting as positive regulators, while HFR1 inhibits these responses (Hornitschek et al., 2009). PIF4 and PIF5 support shade avoidance syndrome by directly binding to G-boxes in promoters of shade marker genes, but their action is limited in the shade when HFR1 accumulates. Since PIF4 and PIF5 act upstream of ABA signaling (Ueda et al., 2020) by directly activating the expression of $A B I 5$ (Sakuraba et al., 2014), the signaling sequence is as follows: HFR1 -\| PIF4/PIF5 $\rightarrow$ ABI5 (Figure 4).
Likewise, via interference with PIF4 by binding to promoters of genes that function in cell elongation, HFR1 regulates cell elongation in response to high temperatures (Ikeda et al., 2021). PIF1 and HFR1 undergo mutual degradation in the dark. Under red and far-red light, HFR1 is stabilized by phytochromemediated inhibition of COP1-SPA. The increased abundance of HFR1 sequesters PIF1 and other PIFs to promote seed germination and seedling de-etiolation under light ( $\mathrm{Xu}$ et al., 2017; Pham et al., 2018b). DET1 suppresses seed germination by destabilizing HFR1 and stabilizing PIF1 (Shi et al., 2015).

In post-germination seedling development, low R:FR conditions induce the accumulation of $\mathrm{ABA}$ and modulate the expression of several ABA biosynthetic and signaling genes (Yadukrishnan and Datta, 2021). ABA induced in response to shade inhibits the shade-triggered hypocotyl elongation by the action of ABI3 and ABI4 (Ortiz-Alcaide et al., 2019). HFR1 does not interact with transcription factors $\mathrm{ABI} 3$ and $\mathrm{ABI} 4$ (BioGRID). Therefore, the role of HFR1 in ABA-induced hypocotyl elongation remains to be studied.

\section{FCA/FLC IN FLOWER DEVELOPMENT AND THERMAL ADAPTATION}

The RNA-binding protein FCA is a component of flowering pathways in Arabidopsis and a regulator of the FLC gene, which encodes MADS-box protein FLOWERING LOCUS C (Tian et al., 2019). FCA interacts with ABI5 and is essential for the proper expression of ABI5-regulated genes involved in antioxidant defense and thermotolerance (Lee et al., 2015). FCA not only regulates the function of many genes involved in adaptation to stress-induced ROS, heat, cold, and drought conditions via FLC and ABI5 but also adjusts the function of protective genes by itself through chromatin modification and RNA metabolism (Lee et al., 2015).

In turn, signaling components of ABA affect FLC expression, thereby influencing floral transition in Arabidopsis. ABA signaling causes the inhibitory effect on floral transition by stimulating the $\mathrm{SnRK} 2 \mathrm{~s} \rightarrow \mathrm{ABI} 5$ (and/or other ABFs) $\rightarrow$ FLC pathway (Wang et al., 2013). Direct binding of ABI5 to the ABRE/G-box promoter elements in FLC was demonstrated by chromatin immunoprecipitation. Shu et al. (2016) showed that ABI4, an important protein in the ABA signaling network, negatively regulates floral transition by directly activating FLC transcription. Interestingly, the authors characterized the observed effect as the "tip of the iceberg" and suggested that further discoveries will be made in this area.

Histone acetylation is important in the FCA-mediated thermal adaptation of developing seedlings, chlorophyll biosynthesis, and seedling photosynthesis ( $\mathrm{Ha}$ et al., 2017). The FLC/FCA module also functions in cold conditions, providing adaptation to winter temperatures through an FLC antisense transcript COOLAIR (Jiao et al., 2019; Tian et al., 2019). It has been shown that FCA interacts with SWI3A and SWI3B, components of the Switch/Sucrose non-fermenting, ATP-dependent chromatin remodeling complex (SWI/SNF CRC; Sarnowski et al., 2005), suggesting the role the chromatin-based control on FCA function. 
The signaling sequence $\mathrm{FCA} \rightarrow \mathrm{ABI} \rightarrow F L C$ (Figure 3) is interesting and needs further examination, especially concerning brassinosteroid signaling and acclimation processes (Bulgakov and Avramenko, 2020; Jing and Lin, 2020). Thus, the FLC/ FCA module is regulated both by the ABA pathway and influences the ABA components, and these two signaling systems jointly coordinate the processes of flower development under different temperature conditions.

\section{ELF3: CIRCADIAN SYSTEM COMPENSATION AGAINST DAILY AND SEASONAL CHANGES}

ELF3 encodes a nuclear protein that is expressed rhythmically and interacts with phyB to regulate plant development and flowering. The important role of this regulator, as a component of the ELF3-ELF4-LUX ARRHYTHMO (LUX) evening complex, is to modulate light input to the circadian clock and connect light signaling with thermotolerance. ELF3 negatively regulates the activity of PIF4 at high temperatures (Sakuraba et al., 2014; Kim et al., 2020), establishing the ELF3-PIF4-ABI5 signaling module. As a result, PIF4 accelerates leaf senescence at high ambient temperature by increasing the expression of the major senescence-promoting NAC transcription factor ORESARA1 with the participation of ABA and ethylene signaling. Although GO annotation indicates that ELF3 is involved in the ABA signaling, the direct interaction of ELF3 (as well as ELF4 and LUX) with ABA signaling components has not been described.

Recent studies of rhythmic chromatin modifications revealed a role of chromatin remodeling factors in ELF3 functioning (Zhao et al., 2021). For instance, histone deacetylase 9 (HDA9) interacts with ELF3 (Lee et al., 2019; Park et al., 2019) and can be considered as an early regulator of thermomorphogenesis. HDA9 is also can interact with many transcription factors, including $\mathrm{HY} 5, \mathrm{ABI} 3$, and $\mathrm{ABI} 4$, thus contributing to the formation of HDA9-dependent epigenetic states regulating growth, acclimation, photoperiodic flowering, senescence, aging, dormancy, and germination (de Rooij et al., 2020). Likewise, SWI/SNF chromatin remodelers interact with many players of the light-ABA network, such as the ABA-related protein phosphatases $\mathrm{ABI} 1, \mathrm{ABI}, \mathrm{HAB} 1$, and PP2CA, SnRK2s, the $\mathrm{ABA}$-related transcription factors $\mathrm{ABI} 3, \mathrm{ABF} 1$, and $\mathrm{ABF} 3$ as well as proteins from the light signaling system: LUX, CO and CONSTANS-like proteins, FCA, the transcription factor SPATULA, and others (Bulgakov et al., 2019). Thus, epigenetic interactions in the ABA-light signaling system represent a promising topic related to the establishment of local epigenetic landscapes.

\section{LIGHT, COLD TOLERANCE, AND SNRK2S}

Above we discussed that HY5 can support the development of cold acclimation, joining cold and light signaling (Section
"HY5-Based Modules"). Transcription factor ICE1 (inducer of $\mathrm{CBF}$ expression 1) represents another important protein in establishing cold resistance. The intersection of the signaling pathways of these cold resistance factors is of great interest for bioengineering purposes. The SnRK2.6/OST1-HOS1-ICE1 signaling module controls cold tolerance via the CBF-COR cold signaling pathway (Ding et al., 2015). In this module, OST1 activates ICE1, and the pleiotropic regulator HOS1 (E3 ubiquitin-protein ligase; synonym: high expression of osmotically responsive genes 1 ) is necessary to adapt plant development both to short-term cold stress and freezing tolerance by interacting with ICE1 (Dong et al., 2006; Ding et al., 2015; Ye et al., 2019). In hos 1 mutants, ICE1 is not degraded (Dong et al., 2006), which leads to the acquisition of cold tolerance via the unified ICE-CBF pathway (Chinnusamy et al., 2003; Kim et al., 2015). ICE1 induces $C B F 1, C B F 2$, and $C B F 3$ by binding to the gene promoters (Kim et al., 2015). The module HOS $1-\|$ ICE $1 \leftarrow$ OST 1 is shown in Figure 5. HOS1 not only inhibits ICE1 but also physically interacts with $\mathrm{PhyB}$ and $\mathrm{CO}$ and suppresses the function of PIF4 (Kim et al., 2017), thereby linking cold response and photoperiodic response (Lazaro et al., 2015). Therefore, HOS1 is controlled by the ABA signaling via SnRK2.6/OST1, and by light signaling via phyB. This creates a question about crossing these two important pathways.

HOS1 and HY5 do not interact physically (BioGRID). The intersection of their signaling occurs via ABI5, as indicated in Figure 5. Rapid local and systemic stomatal responses of Arabidopsis to intense light are dependent on the function OST1/SnRK2.6 (Devireddy et al., 2020). These responses are dependent on ABA-derived ROS signaling by the OST1/SnRK2.6 $\rightarrow$ RbohD pathway (Devireddy et al., 2020). The process of cold acclimation can be realized by RbohD activation at the transcriptional and posttranscriptional levels through ABI5 $\rightarrow$ RbohD and SnRK2.6 $\rightarrow$ RbohD, thus increasing $\mathrm{H}_{2} \mathrm{O}_{2}$ levels, which is necessary to establish cold protection and tolerance to excess light (Zhou et al., 2012; Wang et al., 2018; Devireddy et al., 2020). Considering ROS production as an important factor for cold and high-intensity light acclimation, Miller et al. (2009) and Mittler et al. (2011) showed that RbohD plays a key role in these processes.

The resulting signaling modules for the HOS1 role in these processes can be written as HOS1 - $\|$ ICE1 - $\|$ ABI5 $\rightarrow$ RbohD and HOS1 $-\|$ PIF4 $\rightarrow A B I 5 \rightarrow R b o h D$, which have an overlap in ROS regulation via $A B I 5$ function (Figure 5).

Another way of cold adaptation is the signal crossing between PIF3, CBF1, CBF2, and CBF3 (Figure 6). Jiang et al. (2020) showed that PIF3 interacts with $\mathrm{CBF} 1, \mathrm{CBF} 2$, and $\mathrm{CBF} 3$ proteins. They proposed a model in which $\mathrm{CBF}$ proteins, accumulated in response to cold, interact with PIF3, and this interaction prevents PIF3 and phyB degradation. Cold-stabilized phyB initiates the degradation of PIF1, PIF4, and PIF5, which allows the de-repression of $C O R$ genes. Collectively, these data mean that $C B F 1, C B F 2$, and $C B F 3$ genes are regulated by SnRK2.6-ICE1 and possibly by $A B F 2$ and $A B F 4$ from the side of the ABA signaling, as well as PIF3 and LHY/CCA1 from the side of the light signaling (Figure 6). 


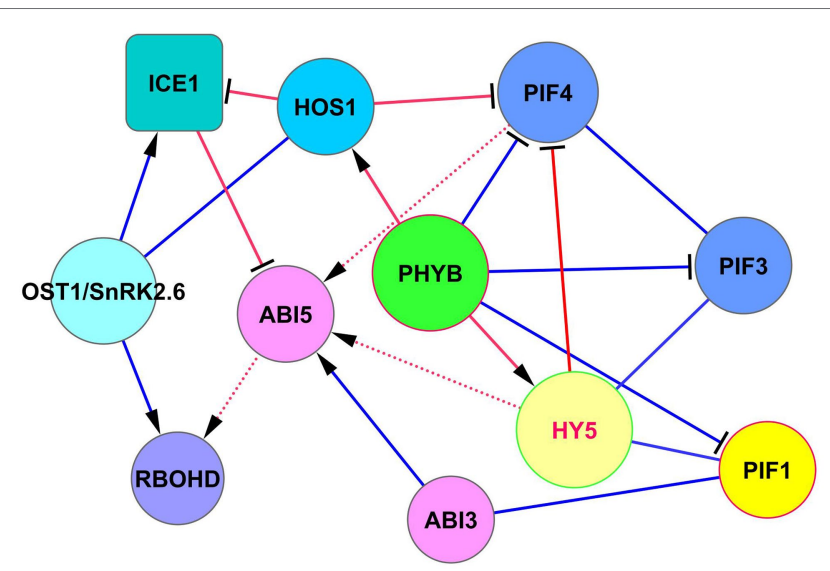

FIGURE 5 | The intersection of ICE1 and HY5 functions in cold adaptation. ABI5 serves as a concentrator of ICE1, PIF4, and HY5 signaling. ABI5 receives signals from these proteins to regulate $R b o h D$ expression and $\mathrm{H}_{2} \mathrm{O}_{2}$ level to establish cold protection. Note, that HY5 does not physically interact with PIF4 (BioGRID) but regulates its function through competitive chromatin binding to PIF4 targets (Gangappa and Kumar, 2017). ICE1 physically associates with $\mathrm{ABI} 5$ and antagonizes its transcriptional function by concurring with G-box type cis elements in promoters of responsive genes (Hu et al., 2019). Abbreviations: ICE1, inducer of CBP expression 1; HOS1, E3 ubiquitin-protein ligase HOS1; ABI3/5, ABA insensitive 3/5; OST1/ SnRK2.6, SNF1-related protein kinase 2.6; PIFs, phytochrome-interacting transcription factors; $\mathrm{PHYB}$, phytochrome phyB; HY5, transcription factor HY5. RBOHD, respiratory burst oxidase homolog protein D (NADPH oxidase RbohD).

Until recently, the interaction of SnRK2s with components of the light signaling network remained elusive. Li et al. (2021) reported the interaction between SnRK2.6 and phyB and showed a negative role of OST1/SnRK2.6 in stomatal opening induced by red light. A snrk2.6 mutant exhibited a significantly larger stomatal aperture under red light treatment, while overexpression lines exhibited smaller stomatal apertures (Li et al., 2021). This observation places ABA signaling, mediated through SnRK2.6, upstream of phyB signaling.

\section{CONCLUDING REMARKS}

The rapidly growing number of articles on the association of ABA and light signaling is driven by the emerging prospects of plant growth management in changing environmental conditions. Regulation of plant development in greenhouses is also important because the ability to manipulate spectral characteristics with LED sources would help combine growth control and stress tolerance. Surprisingly, analysis of protein interaction networking combined with analysis of the literature revealed a relatively small number of interacting modules (Table 1).

These modules are at different stages of understanding their functioning. If crossing of $\mathrm{ABA}$ and light signaling on the $A B I 5$ promoter, ABA-associated HY5- and FHY3/FAR1-based modules relatively well worked out, then LHY/CCA1, ELF3, and FCA modules require further in-depth study. Likewise,

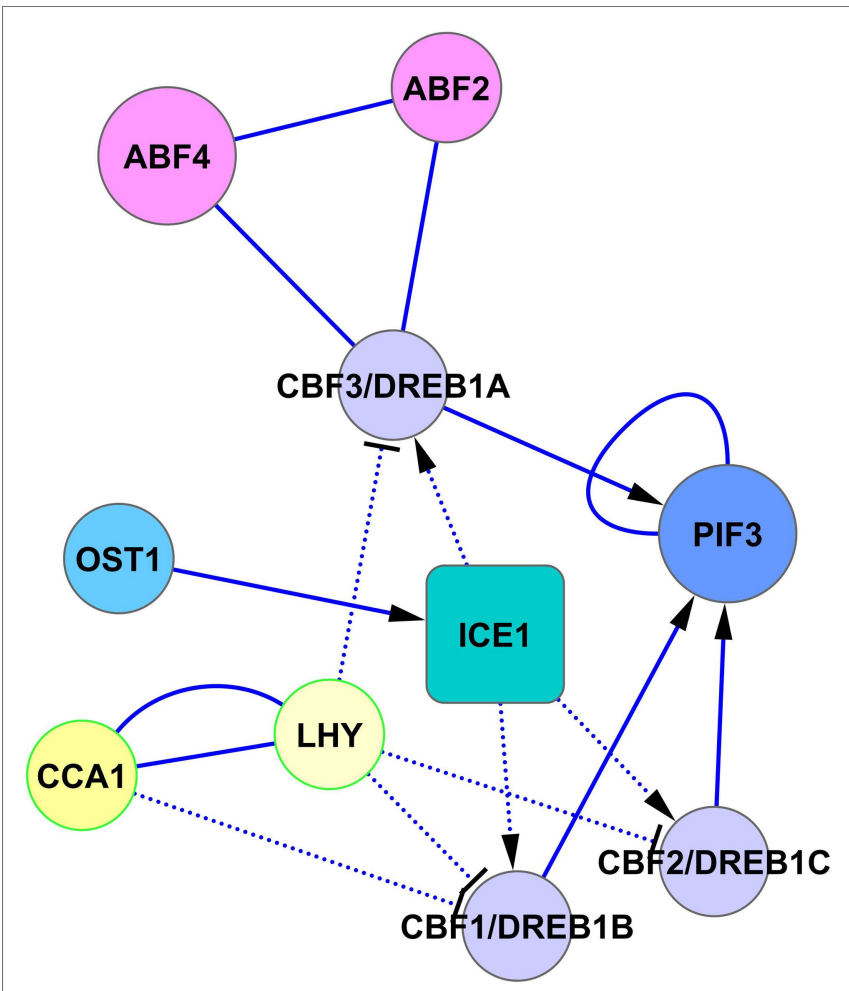

FIGURE 6 | Regulation of CBF/DREB1 pathway by ABA and light signaling. The activated CBF-cold-responsive $(C O R)$ cold-signaling pathway increases cold tolerance. Transcription of CBF1/DREB1B, CBF2/DREB1C, and CBF3/ $D R E B 1 A$ is regulated oppositely by $A B A$ signaling via the cold-activated SnRK2.6-ICE1 pathway and light signaling via CCA1/LHY. CCA1 and LHY suppress the expression of DREB1s under unstressed conditions and are rapidly degraded in response to cold (Kidokoro et al., 2021), allowing activation of the CBF-COR cold signaling pathway. In turn, CBF proteins, accumulated in response to cold, interact with PIF3 and prevent PIF3 degradation with subsequent de-repression of $C O R$ genes. Abbreviations: ABFs, abscisic acid responsive elements-binding factors; PIF3, transcription factor PIF3; OST1/SnRK2.6, SNF1-related protein kinase 2.6; ICE1, inducer of CBP expression 1; CCA1, circadian clock associated 1; LHY, protein late elongated hypocotyl; CBFs/DREBs, dehydration-responsive element-binding proteins.

the functional importance of ABI3-CO, ABF4-FT, and ABI3TOC1 interactions needs further examination. Interaction of important hubs in ABA and light signaling systems, such as SnRK2.6 and phyB, is beginning to be studied and appears to be promising.

During writing this work, we drew attention to the growing body of articles on the epigenetic regulation of the ABA-light signaling system. Indeed, the study of stress memory of plants, a phenomenon through which information on a past stress cue is retained and results in a modified response upon recurring stress, promises great perspectives in the regulation of plant growth, since chromatin regulators instruct time-dependent control of transcription. Concerning the ABA-light signaling system, this topic will undoubtedly be further developed in the coming years.

Summarizing the information described, we would like to highlight several key points. 
- As before, ABI5 is the main protein that binds ABA and light signaling. Recent research has added a new level of complexity to the process mediated by the HY5-ABI5 interaction, since HY5 itself is under complex control. New modules have been added such as DET1 - $\|$ FHY3 $\rightarrow$ ABI5 and DET1/ $\mathrm{COP} 1 \rightarrow \mathrm{PIF} 4 \rightarrow \mathrm{ABI} 5$, and a new look is now needed to assess the overall picture of the impact of light signaling components on ABA signaling mediated through ABI5 to fully understand the logic of cooperation.

- The influence of class 3 sucrose nonfermenting-1-related protein kinases (SnRK2s) on the light signaling system is an important new topic.

- Interaction of ABA receptors with PIFs, because this new topic directly links early ABA signaling to light signaling.

- Part of the research that deals with ROS, as more and more data point to the role of ROS in ABA-light cooperation.

- The FCA $\rightarrow \mathrm{ABI} \rightarrow$ FLC signaling sequence is also a key, because FCA/FLC system is now under deep investigation and FCA/FLC affect ABA signaling and ABA signaling affects FCA/FLC. By itself, FLC significantly reduces plant water use because it binds to multiple target genes involved in the response to water shortage. In turn, FCA regulates the function of many genes involved in adaptation to

\section{REFERENCES}

Adams, S., Grundy, J., Veflingstad, S. R., Dyer, N. P., Hannah, M. A., Ott, S., et al. (2018). Circadian control of abscisic acid biosynthesis and signalling pathways revealed by genome-wide analysis of LHY binding targets. New Phytol. 220, 893-907. doi: 10.1111/nph.15415

Assmann, S. M., and Jegla, T. (2016). Guard cell sensory systems: recent insights on stomatal responses to light, abscisic acid, and CO 2. Curr. Opin. Plant Biol. 33, 157-167. doi: 10.1016/j.pbi.2016.07.003

Belbin, F. E., and Dodd, A. N. (2018). ABA signalling is regulated by the circadian clock component LHY. New Phytol. 220, 661-663. doi: 10.1111/ nph. 15473

Braun, P., Carvunis, A. R., Charloteaux, B., Dreze, M., Ecker, J. R., Hill, D. E., et al. (2011). Evidence for network evolution in an Arabidopsis interactome map. Science 333, 601-607. doi: 10.1126/science.1203877

Brunetti, C., Sebastiani, F., and Tattini, M. (2019). Review: ABA, flavonols, and the evolvability of land plants. Plant Sci. 280, 448-454. doi: 10.1016/j. plantsci.2018.12.010

Bulgakov, V. P., and Avramenko, T. V. (2020). Linking brassinosteroid and ABA signaling in the context of stress acclimation. Int. J. Mol. Sci. 21:5108. doi: $10.3390 / \mathrm{ijms} 21145108$

Bulgakov, V. P., Wu, H. C., and Jinn, T. L. (2019). Coordination of ABA and chaperone signaling in plant stress responses. Trends Plant Sci. 24, 636-651. doi: 10.1016/j.tplants.2019.04.004

Cañibano, E., Bourbousse, C., García-León, M., Gómez, B. G., Wolff, L., García-Baudino, C., et al. (2021). DET1-mediated COP1 regulation avoids HY5 activity over second-site gene targets to tune plant photomorphogenesis. Mol. Plant 14, 963-982. doi: 10.1016/j.molp.2021.03.009

Catalá, R., Medina, J., and Salinas, J. (2011). Integration of low temperature and light signaling during cold acclimation response in Arabidopsis. Proc. Natl. Acad. Sci. U. S. A. 108, 16475-16480. doi: 10.1073/pnas.1107161108

Chai, T., Zhou, J., Liu, J., and Xing, D. (2015). LSD1 and HY5 antagonistically regulate red light induced-programmed cell death in Arabidopsis. Front. Plant Sci. 6:292. doi: 10.3389/fpls.2015.00292

Chen, D., Xu, G., Tang, W., Jing, Y., Ji, Q., Fei, Z., et al. (2013). Antagonistic basic helix-loop-helix/bZIP transcription factors form transcriptional modules that integrate light and reactive oxygen species signaling in Arabidopsis. Plant Cell 25, 1657-1673. doi: 10.1105/tpc.112.104869 stress-induced ROS, heat, cold, and drought through FLC and $\mathrm{ABI}$, and regulates the function of defense genes through chromatin modification.

- Chromatin modifications, because many of the ABA signaling components and light signaling components are under the control of chromatin modifiers. There are currently no review articles describing the ABA-light interaction from this point of view, but new experimental data are constantly being added.

\section{AUTHOR CONTRIBUTIONS}

VB contributed to conception, data analysis, and manuscript writing. OK performed analysis and interpretation of data, revising for important intellectual content, and final approval. All authors contributed to the article and approved the submitted version.

\section{FUNDING}

Financial support was provided by the Russian Science Foundation, Grant No. 20-16-00016 (VB).

Chen, H., Zhang, J., Neff, M. M., Hong, S. W., Zhang, H., Deng, X. W., et al. (2008). Integration of light and abscisic acid signaling during seed germination and early seedling development. Proc. Natl. Acad. Sci. U. S. A. 105, 4495-4500. doi: 10.1073/pnas.0710778105

Chinnusamy, V., Ohta, M., Kanrar, S., Lee, B.-H., Hong, X., Agarwal, M., et al. (2003). ICE1: a regulator of cold-induced transcriptome and freezing tolerance in Arabidopsis. Genes Dev. 17, 1043-1054. doi: 10.1101/gad.1077503

Choi, H., and Oh, E. (2016). PIF4 integrates multiple environmental and hormonal signals for plant growth regulation in Arabidopsis. Mol. Cells 39, 587-593. doi: 10.14348/molcells.2016.0126

Clack, T., Shokry, A., Moffet, M., Liu, P., Faul, M., and Sharrock, R. A. (2009). Obligate heterodimerization of Arabidopsis phytochromes $\mathrm{C}$ and $\mathrm{E}$ and interaction with the PIF3 basic helix-loop-helix transcription factor. Plant Cell 21, 786-799. doi: 10.1105/tpc.108.065227

Courbier, S., and Pierik, R. (2019). Canopy light quality modulates stress responses in plants. iScience 22, 441-452. doi: 10.1016/j.isci.2019.11.035

de Rooij, P. G. H., Perrella, G., Kaiserli, E., and van Zanten, M. (2020). The diverse and unanticipated roles of histone deacetylase 9 in coordinating plant development and environmental acclimation. J. Exp. Bot. 71, 6211-6225. doi: $10.1093 / \mathrm{jxb} / \mathrm{eraa} 335$

Devireddy, A. R., Liscum, E., and Mittler, R. (2020). Phytochrome B is required for systemic stomatal responses and reactive oxygen species signaling during light stress. Plant Physiol. 184, 1563-1572. doi: 10.1104/pp.20.01084

Ding, Y., Li, H., Zhang, X., Xie, Q., Gong, Z., and Yang, S. (2015). OST1 kinase modulates freezing tolerance by enhancing ICE1 stability in Arabidopsis. Dev. Cell 32, 278-289. doi: 10.1016/j.devcel.2014.12.023

Dong, C.-H., Agarwal, M., Zhang, Y. Y., Xie, Q., and Zhu, J.-K. (2006). The negative regulator of plant cold responses, HOS1, is a RING E3 ligase that mediates the ubiquitination and degradation of ICE1. Proc. Natl. Acad. Sci. U. S. A. 103, 8281-8286. doi: 10.1073/pnas.0602874103

Dong, T., Park, Y., and Hwang, I. (2015). Abscisic acid: biosynthesis, inactivation, homoeostasis and signaling. Essays Biochem. 58, 29-48. doi: 10.1042/ bse 0580029

Favero, D. S. (2020). Mechanisms regulating PIF transcription factor activity at the protein level. Physiol. Plant. 169, 325-335. doi: 10.1111/ppl.13075

Fujii, H., Chinnusamy, V., Rodrigues, A., Rubio, S., Antoni, R., Park, S. Y., et al. (2009). In vitro reconstitution of an abscisic acid signalling pathway. Nature 462, 660-664. doi: 10.1038/nature08599 
Gangappa, S. N., and Botto, J. F. (2016). The multifaceted roles of HY5 in plant growth and development. Mol. Plant 9, 1353-1365. doi: 10.1016/j. molp.2016.07.002

Gangappa, S. N., and Kumar, S. V. (2017). DET1 and HY5 control PIF4mediated thermosensory elongation growth through distinct mechanisms. Cell Rep. 18, 344-351. doi: 10.1016/j.celrep.2016.12.046

Gangappa, S. N., and Kumar, S. V. (2018). DET1 and COP1 modulate the coordination of growth and immunity in response to key seasonal signals in Arabidopsis. Cell Rep. 25, 29-37.e3. doi: 10.1016/j.celrep.2018.08.096

Gómez-Ocampo, G., Ploschuk, E. L., Mantese, A., Crocco, C. D., and Botto, J. F. (2021). BBX21 reduces abscisic acid sensitivity, mesophyll conductance and chloroplast electron transport capacity to increase photosynthesis and water use efficiency in potato plants cultivated under moderated drought. Plant J. 108, 1131-1144. doi: 10.1111/tpj.15499

Guo, Z., Xu, J., Wang, Y., Hu, C., Shi, K., Zhou, J., et al. (2021). The phyBdependent induction of HY5 promotes iron uptake by systemically activating FER expression. EMBO Rep. 22:e51944. doi: 10.15252/embr.202051944

Ha, J. H., Kim, J. H., Kim, S. G., Sim, H. J., Lee, G., Halitschke, R., et al. (2018). Shoot phytochrome B modulates reactive oxygen species homeostasis in roots via abscisic acid signaling in Arabidopsis. Plant J. 94, 790-798. doi: $10.1111 /$ tpj. 13902

Ha, J. H., Lee, H. J., Jung, J. H., and Park, C. M. (2017). Thermo-induced maintenance of photo-oxidoreductases underlies plant autotrophic development. Dev. Cell 41, 170-179.e4. doi: 10.1016/j.devcel.2017.03.005

Hornitschek, P., Lorrain, S., Zoete, V., Michielin, O., and Fankhauser, C. (2009). Inhibition of the shade avoidance response by formation of nonDNA binding bHLH heterodimers. EMBO J. 28, 3893-3902. doi: 10.1038/ emboj.2009.306

Hu, Y., Han, X., Yang, M., Zhang, M., Pan, J., and Yu, D. (2019). The transcription factor INDUCER OF CBF EXPRESSION1 interacts with ABSCISIC ACID INSENSITIVE5 and DELLA proteins to fine-tune abscisic acid signaling during seed germination in Arabidopsis. Plant Cell 31, 1520-1538. doi: $10.1105 /$ tpc. 18.00825

Huang, J., Zhao, X., and Chory, J. (2019). The Arabidopsis transcriptome responds specifically and dynamically to high light stress. Cell Rep. 29, 4186-4199.e3. doi: 10.1016/j.celrep.2019.11.051

Ikeda, M., Mitsuda, N., Ishizuka, T., Satoh, M., and Ohme-Takagi, M. (2021). The CIB1 transcription factor regulates light- and heat-inducible cell elongation via a two-step HLH/bHLH system. J. Exp. Bot. 72, 1795-1808. doi: 10.1093/ jxb/eraa567

Jiang, B., Shi, Y., Peng, Y., Jia, Y., Yan, Y., Dong, X., et al. (2020). Cold-induced CBF-PIF3 interaction enhances freezing tolerance by stabilizing the phyB thermosensor in Arabidopsis. Mol. Plant 13, 894-906. doi: 10.1016/j. molp.2020.04.006

Jiao, F., Pahwa, K., Manning, M., Dochy, N., and Geuten, K. (2019). Cold induced antisense transcription of FLOWERING LOCUS C in distant grasses. Front. Plant Sci. 10:72. doi: 10.3389/fpls.2019.00072

Jing, Y., and Lin, R. (2020). Transcriptional regulatory network of the light signaling pathways. New Phytol. 227, 683-697. doi: 10.1111/nph.16602

Kang, X., Xu, G., Lee, B., Chen, C., Zhang, H., Kuang, R., et al. (2018). HRB2 and BBX21 interaction modulates Arabidopsis ABI5 locus and stomatal aperture. Plant Cell Environ. 41, 1912-1925. doi: 10.1111/pce.13336

Kidokoro, S., Hayashi, K., Haraguchi, H., Ishikawa, T., Soma, F., Konoura, I., et al. (2021). Posttranslational regulation of multiple clock-related transcription factors triggers cold-inducible gene expression in Arabidopsis. Proc. Natl. Acad. Sci. U. S. A. 118:e2021048118. doi: 10.1073/pnas. 2021048118

Kim, C., Kim, S. J., Jeong, J., Park, E., Oh, E., Park, Y.-I., et al. (2020). High ambient temperature accelerates leaf senescence via PHYTOCHROMEINTERACTING FACTOR 4 and 5 in Arabidopsis. Mol. Cells 43, 645-661. doi: $10.14348 /$ molcells.2020.0117

Kim, J.-H., Lee, H.-J., Jung, J.-H., Lee, S., and Park, C.-M. (2017). HOS1 facilitates the phytochrome B-mediated inhibition of PIF4 function during hypocotyl growth in Arabidopsis. Mol. Plant 10, 274-284. doi: 10.1016/j. molp.2016.11.009

Kim, Y. S., Lee, M., Lee, J.-H., Lee, H.-J., and Park, C.-M. (2015). The unified ICE-CBF pathway provides a transcriptional feedback control of freezing tolerance during cold acclimation in Arabidopsis. Plant Mol. Biol. 89, 187-201. doi: $10.1007 /$ s11103-015-0365-3
Kuromori, T., Seo, M., and Shinozaki, K. (2018). ABA transport and plant water stress responses. Trends Plant Sci. 23, 513-522. doi: 10.1016/j. tplants.2018.04.001

Kurup, S., Jones, H. D., and Holdsworth, M. J. (2000). Interactions of the developmental regulator $\mathrm{ABI} 3$ with proteins identified from developing Arabidopsis seeds. Plant J. 21, 143-155. doi: 10.1046/j.1365-313x.2000.00663.x

Lau, O. S., and Deng, X. W. (2010). Plant hormone signaling lightens up: integrators of light and hormones. Curr. Opin. Plant Biol. 13, 571-577. doi: 10.1016/j.pbi.2010.07.001

Lazaro, A., Mouriz, A., Pineiro, M., and Jarillo, J. A. (2015). Red light-mediated degradation of CONSTANS by the E3 ubiquitin ligase HOS1 regulates photoperiodic flowering in Arabidopsis. Plant Cell 27, 2437-2454. doi: 10.1105/ tpc. 15.00529

Lee, J., Choi, B., Yun, A., Son, N., Ahn, G., Cha, J.-Y., et al. (2021). Long-term abscisic acid promotes golden2-like1 degradation through constitutive photomorphogenic 1 in a light intensity-dependent manner to suppress chloroplast development. Plant Cell Environ. 44, 3034-3048. doi: 10.1111/ pce. 14130

Lee, S., Lee, H. J., Jung, J. H., and Park, C. M. (2015). The Arabidopsis thaliana RNA-binding protein FCA regulates thermotolerance by modulating the detoxification of reactive oxygen species. New Phytol. 205, 555-569. doi: 10.1111/nph.13079

Lee, K., Mas, P., and Seo, P. J. (2019). The EC-HDA9 complex rhythmically regulates histone acetylation at the TOC1 promoter in Arabidopsis. Commun. Biol. 2:143. doi: 10.1038/s42003-019-0377-7

Legris, M., Nieto, C., Sellaro, R., Prat, S., and Casal, J. J. (2017). Perception and signalling of light and temperature cues in plants. Plant J. 90, 683-697. doi: $10.1111 /$ tpj.13467

Li, D., Fu, X., Guo, L., Huang, Z., Li, Y., Liu, Y., et al. (2016). FAR-RED ELONGATED HYPOCOTYL3 activates SEPALLATA2 but inhibits CLAVATA3 to regulate meristem determinacy and maintenance in Arabidopsis. Proc. Natl. Acad. Sci. U. S. A. 113, 9375-9380. doi: 10.1073/pnas.1602960113

Li, Y.-Z., Zhao, Z.-Q., Song, D.-D., Yuan, Y.-X., Sun, H.-J., Zhao, J.-F., et al. (2021). SnRK2.6 interacts with phytochrome B and plays a negative role in red light-induced stomatal opening. Plant Signal. Behav. 16:1913307. doi: 10.1080/15592324.2021.1913307

Liang, S., Gao, X., Wang, Y., Zhang, H., Yin, K., Chen, S., et al. (2020). Phytochrome-interacting factors regulate seedling growth through ABA signaling. Biochem. Biophys. Res. Commun. 526, 1100-1105. doi: 10.1016/j. bbrc.2020.04.011

Lin, M., Shen, X., and Chen, X. (2011). PAIR: the predicted Arabidopsis interactome resource. Nucleic Acids Res. 39, D1134-D1140. doi: 10.1093/ nar/gkq938

Lumba, S., Toh, S., Handfield, L. F., Swan, M., Liu, R., Youn, J. Y., et al. (2014). A mesoscale abscisic acid hormone interactome reveals a dynamic signaling landscape in Arabidopsis. Dev. Cell 29, 360-372. doi: 10.1016/j. devcel.2014.04.004

Ma, L., and Li, G. (2018). FAR1-RELATED SEQUENCE (FRS) and FRS-RELATED FACTOR (FRF) family proteins in Arabidopsis growth and development. Front. Plant Sci. 9:692. doi: 10.3389/fpls.2018.00692

Miller, G., Schlauch, K., Tam, R., Cortes, D., Torres, M. A., Shulaev, V., et al. (2009). The plant NADPH oxidase RBOHD mediates rapid systemic signaling in response to diverse stimuli. Sci. Signal. 2:ra45. doi: 10.1126/scisignal.2000448

Mittler, R., Vanderauwera, S., Suzuki, N., Miller, G., Tognetti, V. B., Vandepoele, K., et al. (2011). ROS signaling: the new wave? Trends Plant Sci. 16, 300-309. doi: $10.1016 /$ j.tplants.2011.03.007

Negin, B., Yaaran, A., Kelly, G., Zait, Y., and Moshelion, M. (2019). Mesophyll ABA restrains early growth and flowering but does not directly suppress photosynthesis. Plant Physiol. 180, 910-925. doi: 10.1104/pp.18.01334

Oh, E., Kang, H., Yamaguchi, S., Park, J., Lee, D., Kamiya, Y., et al. (2009). Genome-wide analysis of genes targeted by PHYTOCHROME INTERACTING FACTOR 3-LIKE5 during seed germination in Arabidopsis. Plant Cell 21, 403-419. doi: 10.1105/tpc.108.064691

Ortigosa, A., Fonseca, S., Franco-Zorrilla, J. M., Fernández-Calvo, P., Zander, M., Lewsey, M. G., et al. (2020). The JA-pathway MYC transcription factors regulate photomorphogenic responses by targeting HY5 gene expression. Plant J. 102, 138-152. doi: 10.1111/tpj.14618

Ortiz-Alcaide, M., Llamas, E., Gomez-Cadenas, A., Nagatani, A., Martínez-García, J. F., and Rodríguez-Concepción, M. (2019). Chloroplasts 
modulate elongation responses to canopy shade by retrograde pathways involving HY5 and abscisic acid. Plant Cell 31, 384-398. doi: 10.1105/ tpc.18.00617

Paik, I., Fulu Chen, F., Pham, V. N., Zhu, L., Kim, J.-I., and Huq, E. (2019). A phyB-PIF1-SPA1 kinase regulatory complex promotes photomorphogenesis in Arabidopsis. Nat. Commun. 10:4216. doi: 10.1038/s41467-019-12110-y

Paik, I., Kathare, P. K., Kim, J.-I., and Huq, E. (2017). Expanding roles of PIFs in signal integration from multiple processes. Mol. Plant 10, 1035-1046. doi: 10.1016/j.molp.2017.07.002

Park, H. J., Baek, D., Cha, J. Y., Liao, X., Kang, S. H., McClung, C. R., et al. (2019). HOS15 interacts with the histone deacetylase HDA9 and the evening complex to epigenetically regulate the floral activator GIGANTEA. Plant Cell 31, 37-51. doi: 10.1105/tpc.18.00721

Park, H. J., Kim, W. Y., Pardo, J. M., and Yun, D. J. (2016). Molecular interactions between flowering time and abiotic stress pathways. Int. Rev. Cell Mol. Biol. 327, 371-412. doi: 10.1016/bs.ircmb.2016.07.001

Pham, V. N., Kathare, P. K., and Huq, E. (2018a). Phytochromes and phytochrome interacting factors. Plant Physiol. 176, 1025-1038. doi: 10.1104/pp.17.01384

Pham, V. N., Xu, X., and Huq, E. (2018b). Molecular bases for the constitutive photomorphogenic phenotypes in Arabidopsis. Development 145:dev169870. doi: 10.1242/dev.169870

Qi, L., Liu, S., Li, C., Fu, J., Jing, Y., Cheng, J., et al. (2020). PHYTOCHROMEINTERACTING FACTORS interact with the ABA receptors PYL8 and PYL9 to orchestrate ABA signaling in darkness. Mol. Plant 13, 414-430. doi: 10.1016/j.molp.2020.02.001

Rolauffs, S., Fackendahl, P., Sahm, J., Fiene, G., and Hoecker, U. (2012). Arabidopsis COP1 and SPA genes are essential for plant elongation but not for acceleration of flowering time in response to a low red light to far-red light ratio. Plant Physiol. 160, 2015-2027. doi: 10.1104/pp.112.207233

Sakuraba, Y., Jeong, J., Kang, M.-Y., Kim, J., Paek, N.-C., and Choi, G. (2014). Phytochrome-interacting transcription factors PIF4 and PIF5 induce leaf senescence in Arabidopsis. Nat. Commun. 5:4636. doi: 10.1038/ncomms5636

Sarnowski, T. J., Ríos, G., Jásik, J., Swiezewski, S., Kaczanowski, S., Li, Y., et al. (2005). SWI3 subunits of putative SWI/SNF chromatin-remodeling complexes play distinct roles during Arabidopsis development. Plant Cell 17, 2454-2472. doi: $10.1105 /$ tpc. 105.031203

Seung, D., Risopatron, J. P. M., Jones, B. J., and Marc, J. (2012). Circadian clock-dependent gating in ABA signalling networks. Protoplasma 249, 445-457. doi: 10.1007/s00709-011-0304-3

Shi, H., Wang, X., Mo, X., Tang, C., Zhong, S., and Deng, X. W. (2015). Arabidopsis DET1 degrades HFR1 but stabilizes PIF1 to precisely regulate seed germination. Proc. Natl. Acad. Sci. U. S. A. 112, 3817-3822. doi: 10.1073/ pnas. 1502405112

Shu, K., Chen, Q., Wu, Y., Liu, R., Zhang, H., Wang, S., et al. (2016). ABSCISIC ACID-INSENSITIVE 4 negatively regulates flowering through directly promoting Arabidopsis FLOWERING LOCUS C transcription. J. Exp. Bot. 67, 195-205. doi: 10.1093/jxb/erv459

Tang, W., Ji, Q., Huang, Y., Jiang, Z., Bao, M., Wang, H., et al. (2013). FARRED ELONGATED HYPOCOTYL3 and FAR-RED IMPAIRED RESPONSE1 transcription factors integrate light and abscisic acid signaling in Arabidopsis. Plant Physiol. 163, 857-866. doi: 10.1104/pp.113.224386

Tay, S., Hughey, J. J., Lee, T. K., Lipniacki, T., Quake, S. R., and Covert, M. W. (2010). Single-cell NF- $\kappa B$ dynamics reveal digital activation and analogue information processing. Nature 466, 267-271. doi: 10.1038/nature09145

Tian, Y., Zheng, H., Zhang, F., Wang, S., Ji, X., Xu, C., et al. (2019). PRC2 recruitment and $\mathrm{H} 3 \mathrm{~K} 27 \mathrm{me} 3$ deposition at FLC require FCA binding of COOLAIR. Sci. Adv. 5:eaau7246. doi: 10.1126/sciadv.aau7246

Toledo-Ortiz, G., Johansson, H., Lee, K. P., Bou-Torrent, J., Stewart, K., Steel, G., et al. (2014). The HY5-PIF regulatory module coordinates light and temperature control of photosynthetic gene transcription. PLoS Genet. 10:e1004416. doi: 10.1371/journal.pgen.1004416

Ueda, H., Ito, T., Inoue, R., Masuda, Y., Nagashima, Y., Kozuka, T., et al. (2020). Genetic interaction among phytochrome, ethylene and abscisic acid signaling during dark-induced senescence in Arabidopsis thaliana. Front. Plant Sci. 11:564. doi: 10.3389/fpls.2020.00564
Wang, Y., Li, L., Ye, T., Lu, Y., Chen, X., and Wu, Y. (2013). The inhibitory effect of ABA on floral transition is mediated by ABI5 in Arabidopsis. J. Exp. Bot. 64, 675-684. doi: 10.1093/jxb/ers361

Wang, F., Wu, N., Zhang, L., Ahammed, G. J., Chen, X., Xiang, X., et al. (2018). Light signaling-dependent regulation of photoinhibition and photoprotection in tomato. Plant Physiol. 176, 1311-1326. doi: 10.1104/ pp.17.01143

Xu, X., Kathare, P. K., Pham, V. N., Bu, Q., Nguyen, A., and Huq, E. (2017). Reciprocal proteasome-mediated degradation of PIFs and HFR1 underlies photomorphogenic development in Arabidopsis. Development 144, 1831-1840. doi: $10.1242 /$ dev.146936

Xu, D., Li, J., Gangappa, S. N., Hettiarachchi, C., Lin, F., Andersson, M. X., et al. (2014). Convergence of light and aba signaling on the ABI5 promoter. PLoS Genet. 10:e1004197. doi: 10.1371/journal.pgen.1004197

Xu, D., Wu, D., Li, X.-H., Jiang, Y., Tian, T., Chen, Q., et al. (2020). Light and abscisic acid coordinately regulate greening of seedlings. Plant Physiol. 183, 1281-1294. doi: 10.1104/pp.20.00503

Yadukrishnan, P., and Datta, S. (2021). Light and abscisic acid interplay in early seedling development. New Phytol. 229, 763-769. doi: 10.1111/ nph.16963

Yang, Y., and Liu, H. (2020). Coordinated shoot and root responses to light signaling in Arabidopsis. Plant Commun. 1:100026. doi: 10.1016/j. xplc.2020.100026

Ye, K., Li, H., Ding, Y., Shi, Y., Song, C., Gong, Z., et al. (2019). BRASSINOSTEROID-INSENSITIVE2 negatively regulates the stability of transcription factor ICE1 in response to cold stress in Arabidopsis. Plant Cell 31, 2682-2696. doi: 10.1105/tpc.19.00058

Yoshida, T., Christmann, A., Yamaguchi-Shinozaki, K., Grill, E., and Fernie, A. R. (2019). Revisiting the basal role of ABA - roles outside of stress. Trends Plant Sci. 24, 625-635. doi: 10.1016/j.tplants.2019.04.008

Zhang, S., Liu, H., Yuan, L., Li, X., Wang, L., Xu, X., et al. (2021). Recognition of CCA1 alternative protein isoforms during temperature acclimation. Plant Cell Rep. 40, 421-432. doi: 10.1007/s00299-020-02644-7

Zhao, H., Xu, D., Tian, T., Kong, F., Lin, K., Gan, S., et al. (2021). Molecular and functional dissection of EARLY-FLOWERING 3 (ELF3) and ELF4 in Arabidopsis. Plant Sci. 303:110786. doi: 10.1016/j.plantsci.2020.110786

Zhou, J., Wang, J., Li, X., Xia, X. J., Zhou, Y. H., Shi, K., et al. (2014). $\mathrm{H}_{2} \mathrm{O}_{2}$ mediates the crosstalk of brassinosteroid and abscisic acid in tomato responses to heat and oxidative stresses. J. Exp. Bot. 65, 4371-4383. doi: 10.1093/jxb/ eru217

Zhou, J., Wang, J., Shi, K., Xia, X. J., Zhou, Y. H., and Yu, J. Q. (2012). Hydrogen peroxide is involved in the cold acclimation-induced chilling tolerance of tomato plants. Plant Physiol. Biochem. 60, 141-149. doi: 10.1016/j. plaphy.2012.07.010

Zhu, X., Chen, J., Qiu, K., and Kuai, B. (2017). Phytohormone and light regulation of chlorophyll degradation. Front. Plant Sci. 8:1911. doi: 10.3389/ fpls.2017.01911

Conflict of Interest: The authors declare that the research was conducted in the absence of any commercial or financial relationships that could be construed as a potential conflict of interest.

Publisher's Note: All claims expressed in this article are solely those of the authors and do not necessarily represent those of their affiliated organizations, or those of the publisher, the editors and the reviewers. Any product that may be evaluated in this article, or claim that may be made by its manufacturer, is not guaranteed or endorsed by the publisher.

Copyright (c) 2022 Bulgakov and Koren. This is an open-access article distributed under the terms of the Creative Commons Attribution License (CC BY). The use, distribution or reproduction in other forums is permitted, provided the original author(s) and the copyright owner(s) are credited and that the original publication in this journal is cited, in accordance with accepted academic practice. No use, distribution or reproduction is permitted which does not comply with these terms. 